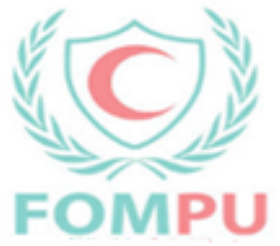

Medicine Updates

Faculty of medicine

https://muj.journals.ekb.eg

dean@med.psu.edu.eg

vice_dean_postgraduate@med.psu.edu.eg

July 2020 ,volume 2 , issue 2

ISSN : 2682-2741

Submitted: $3 / 5 / 2020$

Accepted : 22/5/2020

Pages:15-35

\title{
Clinicopathological and Immunohistochemical Study of High Grade Serous Ovarian Carcinoma
}

Marwa Khashaba, Pathology Department, Faculty of Medicine, Port Said University

Reham Mohamed Nagib, Pathology Department, Faculty of Medicine, Mansoura University

Azza Abdel-Aziz, Pathology Department, Faculty of Medicine, Mansoura University

Ghada Ezzat Eladawei, Pathology Department, Faculty of Medicine, Mansoura University

Mohammed Fawzy Mohamed, Pathology Department, Faculty of Medicine, Mansoura University

\begin{abstract}
:
Background: High grade serous ovarian carcinoma has a heterogenous morphology and marked genomic instability with aggressive behavior and poor overall prognosis. A molecular classification of HGSOC demonstrated 4 subtypes; $\mathrm{C} 1, \mathrm{C} 2, \mathrm{C} 4$ \& C5 as a trial to correlate this molecular classification with patient prognosis. A correlation between these molecular subtypes, clinical data, histopathological criteria and immunohistochemical features needed to be established aiming at individualization of the treatment of each patient to achieve the best outcome.
\end{abstract}


Aim: Investigating for a correlation between the histopathological classification of high grade serous ovarian carcinoma (HGSOC), clinicopathological parameters, and immunohistochemistry.

Methods: Eighty five cases of high grade serous ovarian carcinoma were revised for their clinical data regarding their age, pathological staging, CA 125 levels, ascites, and chemotherapy regimen taken. The cases classified into four groups. Paraffinembedded HGSOC specimens were re-cut at 5 microns thickness sections and stained with haematoxylin and eosin stain and examined for the different histopathological criteria. The tissue sections were also stained immunohistochemically with antibodies against Ki67, CD8, E cadherin, Vimentin, ER \& PR.

Results: HGSOC could be classified into 4 groups (mesenchymal, immunoreactive, Proliferative \& differentiated type either with SET features or papillary features). Ki $67 \&$ CD8 had a strong significant correlation with the proliferative subtype \& immunoreative subtype respectively $(\mathrm{P}<0.001)$. Low $\mathrm{PR}$ expression was also correlated with advanced stage disease in cases underwent 1ry debulking surgery $(\mathrm{P}=0.03)$. in cases received neoadjuvant treatment, differentiated subtype with SET features showed a statistically significant correlation with high CD8 expression.

Keywords: HGSOC, Histopathological classification of HGSOC, IHC of HGSOC.

\section{INTRODUCTION}

Ovarian cancer is the most common lethal gynecological malignancy and is one of major causes of morbidity and mortality among female patients (Berns and Bowtell, 2012). In Egypt, many localized studies conducted at different institutions; of them, studies of Helal et al., 2015 and Nassar et al., 2016 demonstrated that increasing incidence for serous ovarian carcinoma in the Egyptian population is a considerable health problem deserving research. Inability of early diagnosis of the disease is the cause of poor survival and elevated mortality among patients (Narod, 2016). Adding to this, its nonspecific symptoms that mostly overlap with GIT and urinary symptoms diverting the attention of patient and clinician away from the ovary. Additionally, no effective screening strategy is still available despite many trials (Matulonis et al., 2016 and Kurman et al., 2014).

Early study established classification for EOC highlighted 2 distinct types rather than being 2 grades; low grade serous carcinomas (LGSOC) belong to Type (1) while high grade serous carcinomas (HGSC) belong to Type 2 with different molecular pathways involved in pathogenesis of both types. (Shih et al., 2004) HGSOC, which is the dominant and most lethal subtype, are characterized by P53 mutations and marked genomic instability with aggressive behavior, rapid dissemination, late stage at the time of diagnosis and poor overall prognosis (Bowtell et al., 2015 and Kurman and Shih, 2016). 
HGSOC has a heterogeneous morphology; the architecture is characterized by formation of solid masses with slit-like spaces, papillary, glandular or cribriform appearance (Kurman et al., 2014 and Lengyel, 2010). HGSOC cases certainly associated with BRCA1 mutation can exhibit SET architecture pattern (Solid, pseudoEndometrioid and/or Transitional cell carcinoma-like) and contain a greater number of tumor-infiltrating lymphocytes (Soslow et al., 2012).

At the cytological level, HGSOC is characterized by high-grade nuclear atypia; large, hyperchromatic pleomorphic nuclei with multinucleation and prominent eosinophilic nucleoli. High mitotic index > 12/HPF with abundant atypical mitoses. Psammoma bodies are associated with tumors with papillary architecture (Kurman et al., 2014).

The standard treatment is surgical 1ry debulking to remove all the macroscopic disease followed by platinum-based adjuvant chemotherapy. That is the ideal protocol followed with early stage disease (Lawrie et al., 2015). Advanced stage (IIIC or IV) is preferred to receive neoadjuvant chemotherapy (NACT) followed by interval debulking surgery (IDS) (Vergote et al., 2010 and Kehoe et al., 2015).

Gene expression profiling of HGSOC classified ovarian serous carcinomas into 6 clusters from C1-C6 and HGSOC demonstrated four molecular subtypes C1 C2 C4 and $\mathrm{C} 5$; each of them has a significant correlation to patient outcome. These four subtypes were validated in the cancer genome atlas research network (TCGA) study and were named on the basis of gene expression clusters (Tothill et al., 2008, TCGA, 2011).

Mesenchymal subtype (C1) has a cellular stromal reaction and expression of genes of stromal signature and with a poor overall prognosis. Immunoreactive subtype (C2) has tumor infiltrating T-lymphocytes. Its gene expression signature is of immune cell activation with a good overall survival. Differentiated subtype (C4) has low stromal reaction with gene signature closely related to $\mathrm{C} 2$ with a better prognosis. Proliferative subtype (C5) has a gene signature involved in mesenchymal development and found to have a poor overall survival (Testa et al., 2018 and Tothill et al., 2008).

Many studies emerged to find the correlation between histopathological and molecular features in relation to different clinical parameters (Murakami et al., 2016).

There is not yet well-established base regarding this issue. Immunohistochemistry (IHC)could be considered an easy, less expensive and more available method to use in the correlation between histopathology and IHC and help to subtype HGSOC. The current work included different IHC markers including ki67, CD8, E- Cadherin, vimentin, ER and PR. 


\section{MATERIALS \& METHODS}

\section{Sample collection :}

Eighty-five specimens of HGSOC (with available clinical data) were retrieved from archives of surgical pathology lab at Oncology Centre \& University Hospitals; Mansoura University, Egypt during the period from 2010 to 2017. The cases received their treatment at Clinical Oncology and Nuclear Medicine Department. Paraffin blocks of specimens were re-cut at 5 microns thickness and stained with haematoxylin and eosin stain $(\mathrm{H} \& \mathrm{E})$.

\section{Histolopathological classification:}

All $\mathrm{H} \& \mathrm{E}$ stained slides were reviewed to confirm the diagnosis. An algorithm is suggested to categorize cases into 4 subtypes parallel to their molecular classified groups. These included, mesenchymal when cellular stroma is $>10 \%$ of tumor (Murakami et al., 2016), if not, proliferative subtype is considered when mitotic count is >30/10HPF (Popa et al., 2018), if not, then evaluate TILs infiltrating within tumor nests, when are $>$ 20/HPF is classified as immunoreactive (Goode et al., 2017), lastly, according to architectural pattern, is classified as differentiated with SET features that displays $>25 \%$ of tumor morphology SET pattern or differentiated with papillary pattern (Soslow et al., 2012).

\section{Immunohistochemistry:}

_TMA blocks were manually constructed (Foda, 2013 and Shebl et al., 2011), each cases is represented by 3 cores and all cases were subjected to immunohistochemistry with ki67, CD8, E- Cadherin, Vimentin, ER and PR. Immunohistochemical staining was performed on 5 microns sections from formalin-fixed, paraffin-embedded tissue blocks using mouse monoclonal antibodies for all markers, ready to use, (DakoCytomation, Glostrup, Denmark). Positive and negative controls were prepared. Antigen retrieval was performed at $98{ }^{\circ} \mathrm{C}$ in $10 \mathrm{mM}$ sodium citrate buffer $\mathrm{pH} 6$ for 40 minutes. Sections were counterstained with mayer's hematoxylin.

Ki-67 expression is considered high expression when it is expressed in $>25 \%$ of tumor cells, and lower than $25 \%$ was considered low expression (Wang et al., 2016). Figure [5].

Each core was screened for a hotspot of CD8+ TILs at x 20 power, within each hotspot, one high power field at $400 \times$ magnification was evaluated to ensure valid equally comparable areas. Only CD8+ TILs 
within the epithelial component of the tumor (tumor islets) were considered. A score was defined based on CD8+ TIL counts per highpowered field: negative (none), low (1-2 TILs) and high ( $\geq 20$ TILs), similar to the validated method of Zhang and colleagues (Goode et al., 2017). Figure [6].

ER and PR levels evaluated as positive nuclear staining of any intensity in $>10 \%$ of tumor cells as cut off point (Feng et al., 2016). Figure $[7,8]$.

E cadherin immunostaining is interpreted as: $E$ cadherin positive (score $3+$ ) and E-cadherin negative (scores $0,1+$ and $2+$ ) considering $\geq$ $10 \%$ of tumor cells as cut off value. (Miše et al., 2015 and Mohanty et al., 2019). Figure [9].

Vimentin quantitative expression in cancer cells was evaluated as high quantitative vimentin expression when expressed in $>30 \%$ of tumor cells \& low quantitative vimentin expression when expressed in <of $30 \%$ of tumor cells (Szubert et al., 2019). Figure [10].

\section{Statistical methods :}

Data were analyzed using the computer program (SPSS), Version 22 to obtain descriptive statistics. Statistical significance was determined at 95\% level of confidence (i.e. differences will be considered significant if $\mathrm{P}<0.05)$. Qualitative data analysed using Chi-Square test, Monte Carlo test and Fischer Exact test. The point-biserial correlation is used to determine the strength of a linear relationship between one continuous variable and one nominal variable.

\section{RESULTS}

\section{$\underline{\text { Examination of clinical data }}$}

The clinical data of all cases are shown in Table [1]. Considering the treatment protocol groups, there was a statistically significant difference $(\mathrm{P}=0.04)$ between them regarding response to treatment. Table [2] 


\section{Histopathological examination}

The cases are divided according to their histopathological features into 4 groups (mesenchymal subtype, immuoreactive subtype, proliferative subtype and differentiated with SET features \& with papillary features). Table [3]. Figures [1-4]

\section{Correlation between histopathological and clinical data}

No statistically significant correlation found between any of the histopathological subtypes and the clinical data. Table [4].

Correlation between Immunohistochemical data $\mathbb{\&}$

\section{clinicopathological parameters for cases underwent 1ry debulking}

We found a statistically significant correlation between immunoreactive subtype and high CD8 expression $(\mathrm{P}<0.001)$. Additionally, there was a statistically significant correlation between high Ki67 and proliferative subtype. Also, high ER was significantly correlated with proliferative subtype $(\mathrm{P}=0.04)$. Low $\mathrm{PR}$ expression was also correlated with advanced stage disease $(\mathrm{P}=0.03)$. Vimentin expression didn't show correlation with any histopathological parameter. Table [5].

\section{Correlation between Immunohistochemical data\&clinicopathological}

\section{parameters for cases underwent interval debulking surgery}

A statistically significant correlation was found between immunoreactive subtype and high CD8 expression $(\mathrm{P}<0.001)$. Also, there was a statistically significant correlation between high Ki67 and proliferative subtype. Differentiated subtype with SET features showed a statistically significant correlation with high CD8 expression. Table [6].

\section{DISCUSSION}

HGSOC is one of the tumors that unusually to have a good prognosis. Morphologically, no histopathological features of HGSOC 
could be identified associated with good prognosis. Sparse studies are available about the association between HGSOC morphology and molecular features with the prognosis.

The correlation between the histopathological subtypes and the clinical data of HGSOC can predict the subtypes that have the earliest stage at diagnosis and the best behavior. That helps the clinician to stratify patients to give individualized treatment regimens.

The Australian Ovarian Cancer Study Group defined four molecular subtypes of HGSOC (C1, C2, C4\& C5) by gene expression analysis (Tothill et al., 2008) and validated in the TCGA project (Cancer Genome Atlas Research Network, 2011 and Verhaak et al., 2013).

The study of Murakami et al., 2016 described histopathological based four subtypes of HGSOC: mesenchymal transition (MT), immune reactive (IR), solid and proliferative (SP), and papilloglandular (PG). These histopathological subtypes had a significant relation with the previously mentioned molecular studies and have an impact on their prognosis; IR subtype had the best PFS and OS, while MT had the worst prognosis (Murakami et al., 2016 and Darb-Esfahani et al., 2018).

\section{Histopathological data (types) of the studied cases.}

According to the detailed histopathological features described in our study, cases were subdivided into 4 groups including mesenchymal type represented by $29 \%$ of cases, immune reactive type that included $16.5 \%$ of cases ,proliferative type which included other $16.5 \%$, and differentiated type represented by 32 cases; $15 \%$ of them had SET features \& $22 \%$ had papillary architectural features. 
In studies of Murakami et al, 2016, they supposed an algorithm for establishment of histopathological based classification of HGSOC and correlated them with the gene signatures, out of 132 cases; 36\% (MT), $26 \%$ (IR), 24\% (SP) and 14\% (PG). Also in the study of Ohsuga et al., 2017, out of their studied 65 cases included 26\% IR, 10\% PG, 21\% SP, and $41 \%$ MT histopathological subtypes. The previously mentioned studies correlated their cases with certain gene signatures, but we aimed to correlate them with immunohistochemical staining as an inexpensive way to help in expecting the behavior of the disease.

Earlier study demonstrated a histopathological subtype of HGSOC which is with SET features, 24 cases out of 43 cases and correlates significantly with TILs \& state of BRCA1 mutation (Soslow et al., 2012). A recent study of Darb- Esfahani and coworkers classified their studied cases based on their morphology into cases with papillary features (classic type) \& cases with SET features (Darb- Esfahaniet al., 2018).

\section{Correlation between histopathological data (types) and clinical data.}

On correlation between the histopathological subtypes and clinical data, we observed most of our studied cases were diagnosed at an advanced stage (III\&IV) especially in mesenchymal \& proliferative types but without statistically significant association. However, in the reports of Murakami et al, 2016 (on 132 cases of HGSOC) \& Ohsuga et al., 2017 (on 65 cases of HGSC), the mesenchymal type cases were diagnosed significantly in an advanced stage. The study of Ohsuga et al., 2017 added to their study imaging analysis like ( $\mathrm{CT} \& \mathrm{MRI})$ to provide accurate diagnosis as the biopsing of deep located tumors is difficult.

In our study we tried to find a significant role of ascetic fluid (malignant or reactive) in between the different histopathological types; 
out of studied 85 cases, 57 cases had ascites with no significant correlation with the histopathological subtypes. On the other hand, Ohsuga et al., studied the amount of ascites by MRI in different histopathological subtypes and found them significantly in small amount with IR subtypes \& in large amount in MT subtypes, but they didn't demonstrate either ascites was reactive or malignant (Ohsuga et al., 2017).

\section{Treatment response among studied cases}

It is known that NACT is given for patients who have wide disease dissemination or at high risk of perioperative complications (Leary et al., 2016).

In our study, a significant difference observed between patients received NACT and patients not regarding their response to treatment. It was observed that most cases with 1ry debulking surgical procedure were sensitive to treatment but developed recurrence or metastasis of the disease after $>6$ months from date of last cycle, while cases received NACT developed progression in $<6$ months of the last adjuvant cycle.

Majority of HGSOC are chemosensitive and rapidly shrink with initial cycles of NACT asuming patient cure. But it was observed the opposite in some of these cases; relapse occurs in a year after the last adjuvant cycle (Ivantsov et al., 2018).

Recent study of Sokolenko et al provided an explanation for these treatment outcomes. They referred the cause to the difference of the pattern of BRCA1 gene mutation before and after platimun based NACT (Sokolenko et al., 2017). 


\section{Immunohistochemical data \& clinicopathological parameters for cases underwent 1ry debulking.}

$\mathrm{Ki}-67$ is a nuclear antigen used as an indicator of cell proliferation expressed during G1, S, M and G2 phases of cell cycle \& absent in the quiescent non proliferating cell. The Ki-67 highly expressed in HGSOC in relation to LGSOC. That high expression is indicative of high proliferation rate and aggressiveness of tumor cells (Verma et al., 2017 and Laishram et al., 2019).

The current study concluded that Ki-67 expression is highly statistically significant correlated with the proliferative subtype in cases performed 1ry debulking and that is consistent with study of Popa and colleagues when found that mean Ki-67 expression was higher for their studied HGSOC cases and correlated with the high mitotic count >30/ HPF (Popa et al., 2018).

Many studies performed to detect the role of TILs in subtypes of ovarian cancers (Kandalaft et al., 2011). TILs can be assessed through both histopathological and immunohistochemical examinations. CD8 is a valuable marker for TILs (Santoiemma et al., 2015).

In our study, CD8 positivity was found to have a strong statistically significant correlation with immune reactive subtype (either in cases received NACT or not). That is consistent with the findings of Murakami et al, 2016 who studied both intraepithelial \& stromal CD8 positive lymphocytes and found them correlating significantly with immunoreactive type.

Also, the report by Rojas and work group included 50 cases of different malignant surface epithelial ovarian tumors included 10 HGSOC and found a statistically significant correlation between HGSOC type \& 
CD8 positive intratumoral infiltrating lymphocytes, but they didn't establish a histopathological classification of those HGSOC cases (Rojas et al., 2019).

Limited clinical information is available about the possible effects of estrogen and progesterone in ovarian cancer initiation, metastasis and recurrence. Many in vivo \& in vitro trials have been mentioned in many earlier studies about this issue (Modugno et al., 2012). Estrogen \& progesterone induce their effect through complex interaction with receptors (ER \& PR) and induce proliferation, but it also can enhance apoptosis of ovarian cancer cells (Modugno et al., 2012).

It becomes essential to study the role of hormone receptors in HGSOC to give a chance to introduce new treatment modalities.

Our results supposed that low PR expression in tumor cells is associated significantly with advanced stage of the disease (III \& IV). Many studies were in contrast; Feng et al. suggested PR+ group was associated with advanced stage (Feng et al., 2016). Perhaps that discrepancy because we have increased PR negatively stained cases 38 cases and most of them are in advanced stage as most of our studied cases do. In addition to that number of cases in our current study is 85 compared to larger number in the mentioned study.

We also found a significant association between expression of ER \& ki67 and that also proved by Popa et al. in their trial when most of their HGSOC cases are positive for ki67 index > 30/10HPF and also are positive for ER status, but they didn't make a correlation between them (Popa et al., 2018). 


\section{Immunohistochemical data \& clinicopathological parameters for cases underwent interval debulking surgery.}

NACT is given for patients have wide disease dissemination or at high risk of perioperative complications, so the 1ry debulking couldn't be done safely or can get required benefit (Leary et al., 2016).

In the current study, cases received NACT (39 cases), a significant correlation suggested between CD8 positive TILs \& IR subtype (> 20 lymphocytes/HPF) this is consistent with the study of Darb-Esfahani et al. which included HGSOC received neoadjuvant chemotherapy (platinum based either combined with carboplatin or others). Their cases molecularly proved to be IR subtype. They found $>50 \%$ of patients with this subtype are long term survivors with PFS > five years and characterized by intratumoral enrichment of CD8 positive $\mathrm{T}$ cells. However, the study didn't use the histopathology as a basis for classification of their studied cases (Darb-Esfahani et al., 2018).

The current study suggested that differentiated subtype with SET features showed a statistically significant correlation with high CD8 expression in tumor cells. An earlier report described the same when 43 HGSOCs studied as part of TCGA project to find a significant relationship between HGSOC morphology, immunohistochemistry and also added the correlation with the state of BRCA genes. They found the differentiated morphological pattern with SET features has a significant association with diffusely dense TILs in most cases of BRCA1 mutation. Adding to that the current study found that correlation in cases received NACT but the mentioned study not. Also, we tried to identify which T lymphocytes subpopulation by using CD8 IHC which is correlated 
significantly with presence of SET features but the mentioned study didn't (Soslow et al., 2012).

Also, we demonstrated $85 \%$ of cases received NACT have strong significant correlation between low ki67 staining and decrease mitotic figures $<30 / 10 \mathrm{HPF}$ histologically. We also found the mitotic count is significantly decreased $<30 / 10 \mathrm{HPF}$ in relation to cases that not received NACT. That is concomitant with studies of Khandakar et al. and Miller et al. when found a significant lower Ki-67expression after NACT along with significant differences in the tumor morphology (Miller et al. 2008and Khandakar et al. 2014).

Study by Miller and coworkers revealed a reduction in ki67 labelling index in cases displaying significant changes in tumor morphology following NACT (Miller et al. 2008).

Through the mentioned criteria, Ki-67 can be used as therapeutic target in HGSOC treatment through drugs act by inactivation of the proliferation marker will lead to cell death specifically in proliferating cells and thus could be a potential strategy for the treatment.

\section{CONCLUSION \& RECOMMENDATION}

HGSOC is the most lethal gynecological malignancies with a marked molecular instability and heterogenous molecular profile. Histopathological classification of HGSOC and correlation with clinical data and immunohistochemical features could be a helpful method to stratify patients for giving an individualized treatment to patient and predicting their response to treatment modalities. Further studies are needed including larger number of studied cases with correlation of the histopathological features with more clinical and prognostic data. 


\section{REFERENCES}

- $\quad$ Berns E M and Bowtell D D. (2012). The changing view of high-grade serous ovarian cancer. Cancer Research, 72(11), 2701-2704.

- $\quad$ Bowtell D.D, Böhm S, Ahmed A.A et al. (2015). Rethinking ovarian cancer II: Reducing mortality from high-grade serous ovarian cancer. Nature Reviews Cancer, 15(11), 668-679.

- Darb-Esfahani S, Kolaschinski I, Trillsch F, et al. (2018). Morphology and tumour-infiltrating lymphocytes in high-stage, high-grade serous ovarian carcinoma correlated with long-term survival. Histopathology, 73(6),10021012.

- Feng Z, Wen H, Bi R, et al. (2016). A clinically applicable molecular classification for high-grade serous ovarian cancer based on hormone receptor expression. Scientific Reports, 6, 25408.

- Feng Z, Wen H, Bi R, et al. (2016). A clinically applicable molecular classification for high-grade serous ovarian cancer based on hormone receptor expression. Scientific Reports, 6, 25408.

- $\quad$ Foda A. (2013). No- cost manual method for preparation of tissue microarrays having high quality comparable to semiautomated method. Applied Immunohistochemistry \& Molecular Morphology, 21(3), 271-274.

- $\quad$ Goode EL, Block MS, Kalli KR, et al. (2017). Dose-Response Association of CD8+ Tumor-Infiltrating Lymphocytes and Survival Time in High-Grade Serous Ovarian Cancer. JAMA Oncology, 3(12), e173290.

- Helal T, Salman M and Ezz-Elarab S. (2015). Pathology Based Cancer Registry 2001-2010 Ain Shams Faculty of Medicine Cairo, Egypt.

- Ivantsov AO. (2018). Pathological response of ovarian cancer to neoadjuvant chemotherapy. Chinese Clinical Oncology,7(6),59.

- $\quad$ Kandalaft LE, Powell Jr DJ, Singh N, et al. (2011). Immunotherapy for ovarian cancer: what's next?. Journal of Clinical Oncology, 29(7), 925-933.

- $\quad$ Kehoe S, Hook J, Nankivell M, et al. (2015). Primary chemotherapy versus primary surgery for newly diagnosed advanced ovarian cancer (CHORUS): An open-label, randomised, controlled, non-inferiority trial. Lancet, 386(9990), 249-257. 
- Khandakar B, Mathur SR, Kumar L, et al., (2014). Tissue biomarkers in prognostication of serous ovarian cancer following neoadjuvant chemotherapy. Biomed Research International, 2014,401245

- Kurman R.J and Shih Ie M. (2016). The Dualistic Model of Ovarian Carcinogenesis: Revisited, Revised and Expanded. American Journal of Pathology, 186(4), 733-747.

- Kurman RJ, Carcangiu ML, Herrington CS, et al. (2014). WHO classification of tumors of female reproductive organs. 4th ed. Lyon: IARC Press.

- Laishram S, Gupta V, Bhake A, et al. (2019). To assess the utility of proliferative marker Ki-67 in surface epithelial ovarian tumor. Journal of Datta Meghe Institute of Medical Sciences University 14,6-10.

- $\quad$ Lawrie TA, Winter-Roach BA, Heus P, et al. (2015). Adjuvant (postsurgery) chemotherapy for early stage epithelial ovarian cancer. Cochrane Database of Systemic Reviews,2015, (12): CD004706.

- Leary A, Cowan R, Chi D, et al. (2016). Primary Surgery or Neoadjuvant Chemotherapy in Advanced Ovarian Cancer: The Debate Continues.... American Society of Clinical Oncology educational book. American Society of Clinical Oncology. Annual Meeting, 35,153-162.

- Lengyel E. (2010). Ovarian cancer development and metastasis. American Journal of Pathology, 177 (3), 1053-1064.

- Matulonis UA, Sood AK, Fallowfield L, et al. (2016). Ovarian cancer. Nature Reviews Disease Primers, 2, (16061).

- Miller K, Price JH, Dobbs SP, et al. (2008). An immunohistochemical and morphological analysis of post-chemotherapy ovarian carcinoma. Journal of Clinical Pathology, 61(5), 652-657.

- Miše BP, Telesmanić VD, Tomić S, et al. (2015). Correlation Between Ecadherin Immunoexpression and Efficacy of First Line Platinum-Based Chemotherapy in Advanced High Grade Serous Ovarian Cancer. Pathology Oncology Research, 21(2), 347-356.

- $\quad$ Modugno F, Laskey R, Smith AL, et al. (2012). Hormone response in ovarian cancer: time to reconsider as a clinical target?. Endocrine Related Cancer, 19(6), 255-279. 
- Mohanty SK, Tiwari A, Singh C et al. (2019). High-grade ovarian serous carcinomas: Significant correlation of histologic patterns with IMP3 and ECadherin predicting disease recurrence and survival. Annals of Diagnostic Pathology, 40,30-39

- Murakami R, Matsumura N, Mandai M et al. (2016). Establishment of a Novel Histopathological Classification of High-Grade Serous Ovarian Carcinoma Correlated with Prognostically Distinct Gene Expression Subtypes. American Journal of Pathology, 186 (5), 1103-1113.

- Narod S. (2016). Can advanced-stage ovarian cancer be cured?. Nature Reviews and Clinical Oncology, 13(4), 255-261.

- Nassar HR, Sallam Y A, Darwish T, et al. (2016). Clinicopathological, Epidemiologic Characteristics and Treatment Outcomes of Ovarian Cancer Patients at NCI, Cairo University. EC cancer, 2(3), 106-120.

- Ohsuga T, Yamaguchi K, Kido A et al. (2017). Distinct preoperative clinical features predict four histopathological subtypes of high-grade serous carcinoma of the ovary, fallopian tube, and peritoneum. BMC Cancer, 17(1),580.

- $\quad$ Popa M, Corobea A, Munteanu O, et al. (2018). High-Grade versus low grade serous carcinoma of the ovary - current differential diagnosis and prespectives. Archives of the Balkan Medical Union, 53(4), 557-562.

- Rojas K, Prado-Vazquez G, Barcena C, et al. (2019). Tumor-infiltrating Lymphocytes Expression in Stage IIIc/IV of High-grade Serous Ovarian Cancer: Variation with Neoadjuvant Chemotherapy and Prognostic Value. Oncogen 2(2), 8.

- $\quad$ Santoiemma PP and Powell Jr DJ. (2015). Tumor infiltrating lymphocytes in ovarian cancer. Cancer Biology and Therapy, 16(6), 807-820.

- $\quad$ Shebl A M., Zalata K R., Amin M M., et al. (2011). An inexpensive method of small paraffin tissue microarrays using mechanical pencil tips. Diagnostic Pathology, 6, 117.

- $\quad$ Shih Ie M and Kurman RJ. (2004). Ovarian tumorigenesis: A proposed model based on morphological and molecular genetic analysis. The American Journal of Pathology, 164, (5),1511-1518.

- Sokolenko AP, Savonevich EL, Ivantsov AO, et al. (2017). Rapid selection of BRCA1-proficient tumor cells during neoadjuvant 
therapy for ovarian cancer in BRCA1 mutation carriers. Cancer Letters, 397,127-132.

- Soslow RA, Han G, Park KJ, et al. (2012). Morphologic patterns associated with BRCA1 and BRCA2 genotype in ovarian carcinoma. Modern Pathology, 25(4), 625-636.

- $\quad$ Soslow RA, Han G, Park KJ, et al. (2012). Morphologic patterns associated with BRCA1 and BRCA2 genotype in ovarian carcinoma. Modern Pathology, 25(4), 625-636.

- $\quad$ Szubert S, Koper K, Dutsch-Wicherek et al. (2019). High tumor cell vimentin expression indicates prolonged survival in patients with ovarian malignant tumors. Ginekologia Polska 90(1),11-19.

- Testa U, Petrucci E, Pasquini L, et al. (2018). Ovarian Cancers: Genetic Abnormalities, Tumor Heterogeneity and Progression, Clonal Evolution and Cancer Stem Cells. Medicines (Basel), 5(1), 16.

- Tothill RW, Tinker AV, George J, et al. (2008). Novel molecular subtypes of serous and endometrioid ovarian cancer linked to clinical outcome. Clinical Cancer Research, 14 (16), 5198-5208.

- Vergote I, Tropé CG, Amant F, et al. (2010). Neoadjuvant chemotherapy or primary surgery in stage IIIC or IV ovarian cancer. New England Journal of Medicine, 63(10), 943-953.

- Verhaak RG, Tamayo P, Yang JY, et al. (2013). Prognostically relevant gene signatures of high-grade serous ovarian carcinoma. Journal of Clinical Investigation, 123(1), 517-525.

- Verma R, Gupta P, Tiwari N, et al. (2017). Histological grade, CA-125 Levels and IHC expression of ER/PR, HER-2/NEU, p53 and Ki-67 markers in epithelial ovarian neoplasms: A correlative study. International Journal of Advanced Research 5(6), 235-254.

- Wang K, Li D and Sun L. (2016). High levels of EGFR expression in tumor stroma are associated with aggressive clinical features in epithelial ovarian cancer. Oncology Targets and Therapy, 9, 377-386 


\section{TABLES}

Table [1]: Clinical data of studied cases (no. 85)

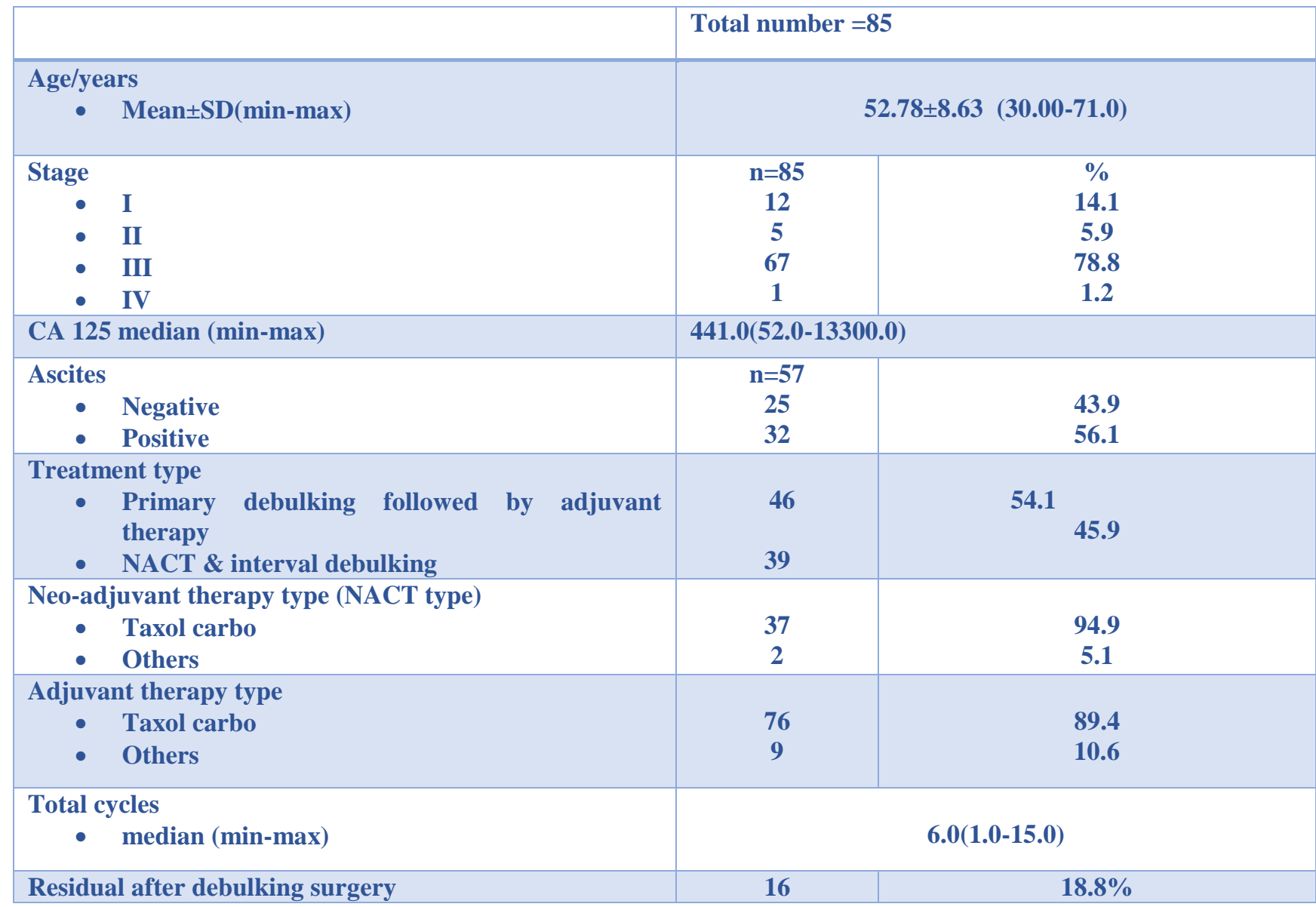

Table (2): Treatment response among studied cases

\begin{tabular}{|c|c|c|c|}
\hline Treatment response & $\begin{array}{c}\mathrm{N}=46(\%) \\
\text { 1ry debulking }\end{array}$ & $\begin{array}{c}\mathrm{N}=39(\%) \\
\text { NACT \& IDS }\end{array}$ & Test of significance \\
\hline - Regression & $14(30.4)$ & $7(17.9)$ & \multirow{3}{*}{$\begin{array}{l}\square^{2}=8.12 \\
\mathrm{P}=0.04 *\end{array}$} \\
\hline $\begin{array}{l}\text { Recurrence } \\
\text { - Metastasis }\end{array}$ & $\begin{array}{l}16(34.8) \\
11(23.9)\end{array}$ & $\begin{array}{c}12(30.8) \\
6(15.4)\end{array}$ & \\
\hline - Progression & $5(10.9)$ & $14(35.9)$ & \\
\hline
\end{tabular}

NACT: neoadjuvant chemotherapy, IDS: interval debulking surgery

$\square^{2}$ :Chi-Square test *statistically significant 
Table (3): Correlation of mitotic activity and treatment protocol

\begin{tabular}{|l|c|c|c|}
\hline Histopathology & $\begin{array}{c}\text { Group 1:1ry } \\
\text { debulking } \\
\text { N= 46(\%) }\end{array}$ & $\begin{array}{c}\text { Group 2: } \\
\text { interval } \\
\text { debulking } \\
\text { N=39(\%) }\end{array}$ & $\begin{array}{c}\text { Test of } \\
\text { significance }\end{array}$ \\
\hline $\begin{array}{l}\text { Mitosis } \\
\text { <30/10 HPF }\end{array}$ & $\begin{array}{l}\text { 20(43.5) } \\
\text { >30/10 HPF }\end{array}$ & $\begin{array}{c}33(84.6) \\
6(15.4)\end{array}$ & $\square^{2}=15.22$ \\
\hline
\end{tabular}




\section{Figures}

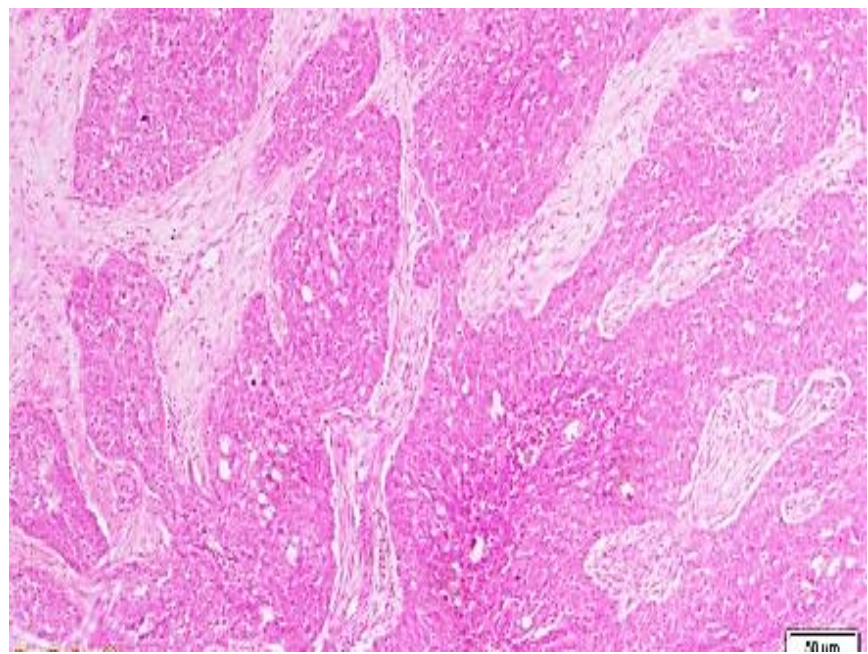

Fig. (1): HGSOC. Cellular stroma. (H\&E X 40).

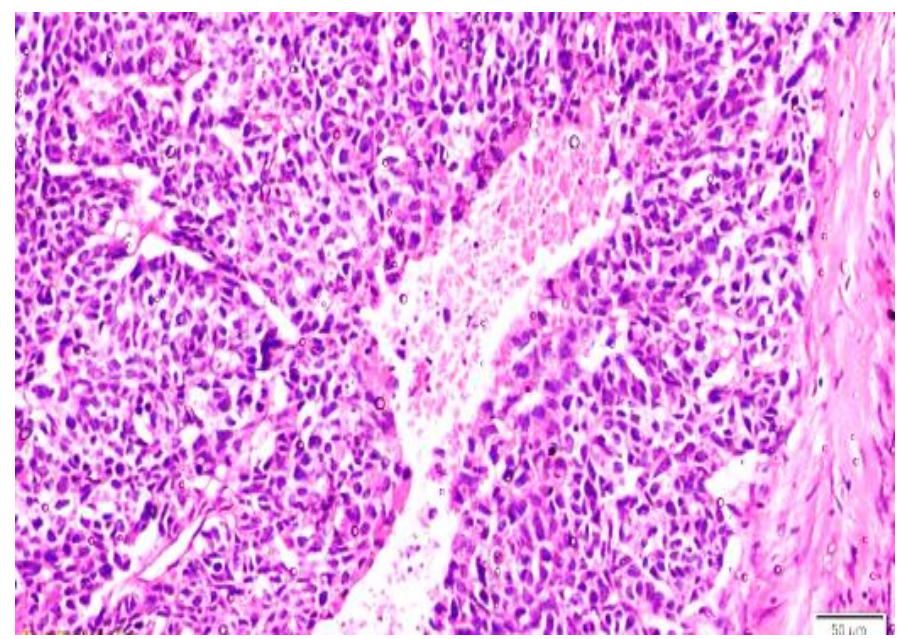

Fig. (2): HGSOC. Differentiated type with SET features $(H \& E X 100)$.

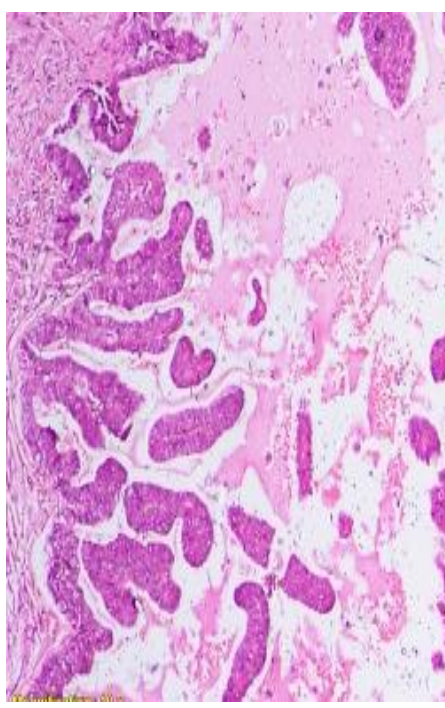

Fig. (3): HGSOC. Differentiated micropapillary architecture. (H\&E X 40).

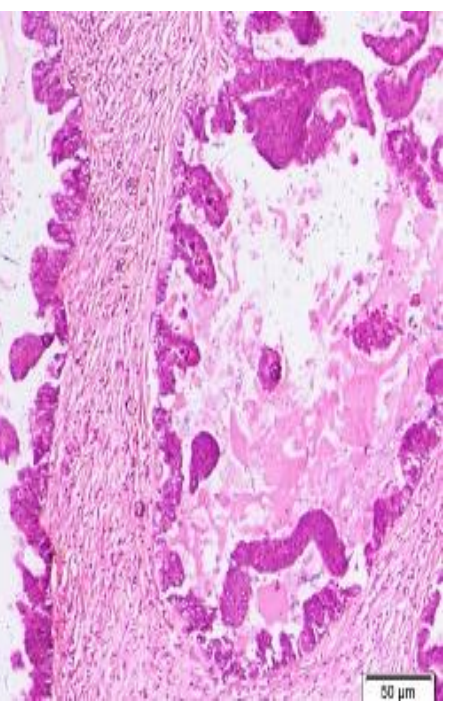

type.

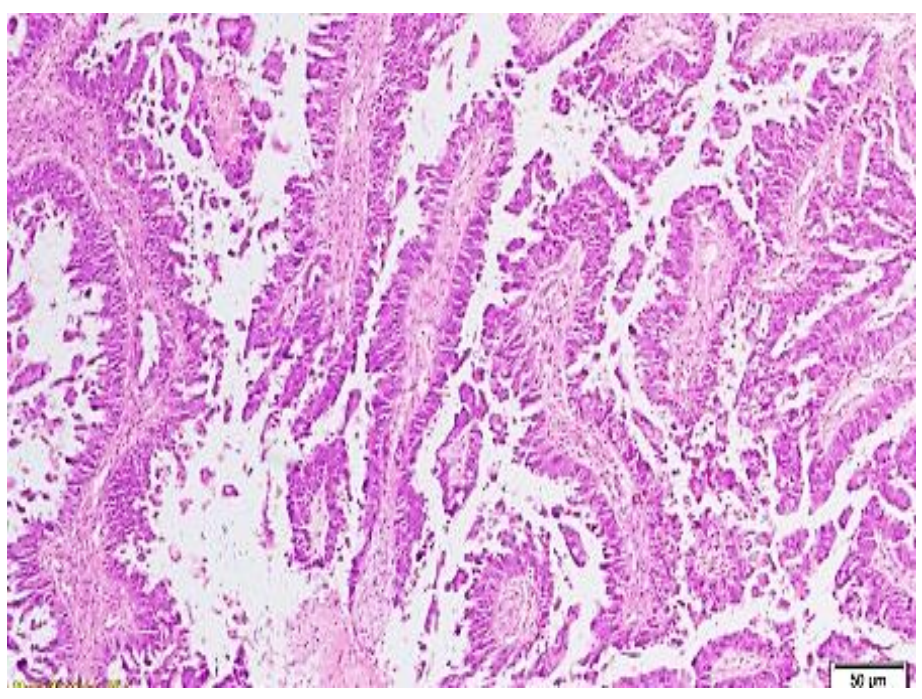

Fig. (4): HGSOC. Differentiated type. Papillary architecture. (H\&E X 40). 


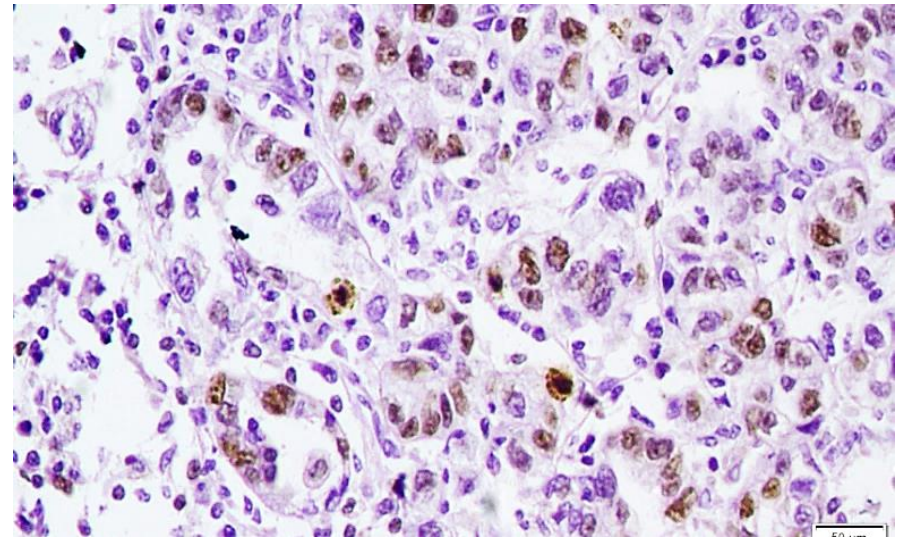

Fig. (5): HGSOC. Positive Ki67 nuclear staining in $>\mathbf{2 5 \%}$ of tumor cells. (Immunohistochemistry (IHC) $\mathrm{X}$ 200)

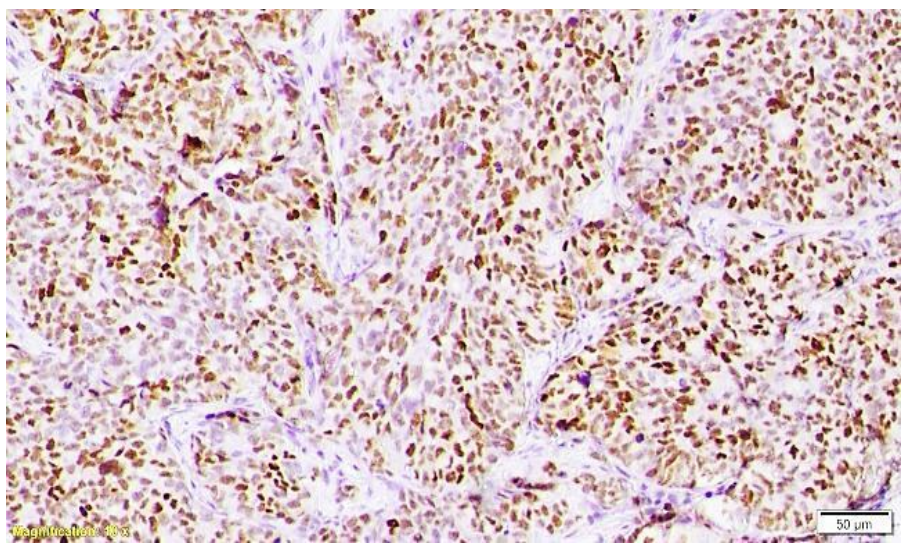

Fig. (7): HGSOC. Positive ER nuclear staining in $>10 \%$ of tumor cells. (Immunohistochemistry (IHC) X 100).

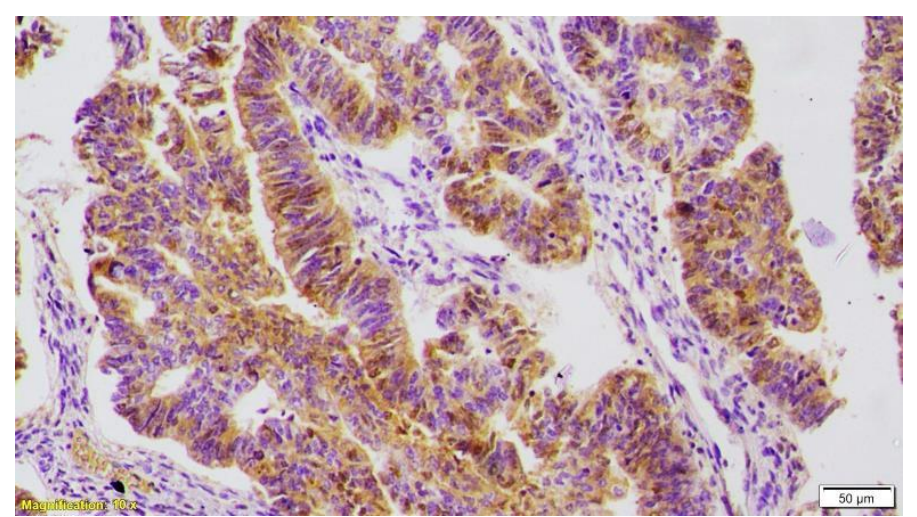

Fig. (9): HGSOC. Positive E Cadherin cytoplasmic staining in $>10 \%$ of tumor cells. (Immunohistochemistry (IHC) X 100).

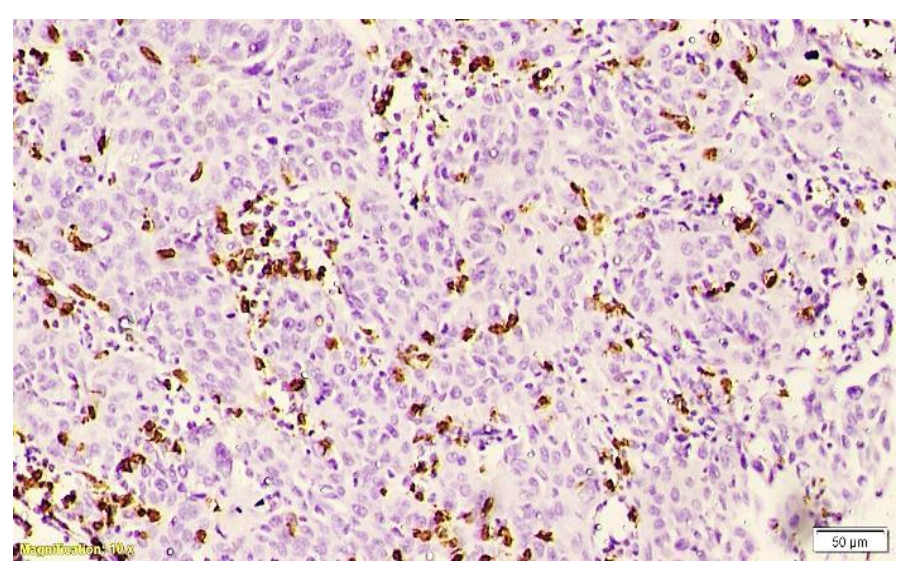

Fig. (6): HGSOC. Positive CD8 membranous staining in >20/HPF. (Immunohistochemistry (IHC) X 100).

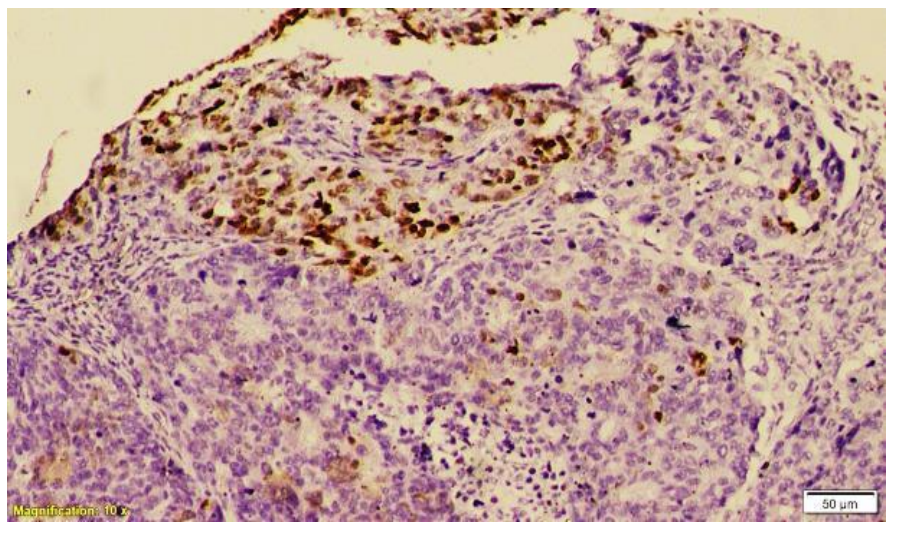

Fig. (8): HGSOC. Positive PR nuclear staining in $>10 \%$ of tumor cells. (Immunohistochemistry (IHC) $X$ 100).

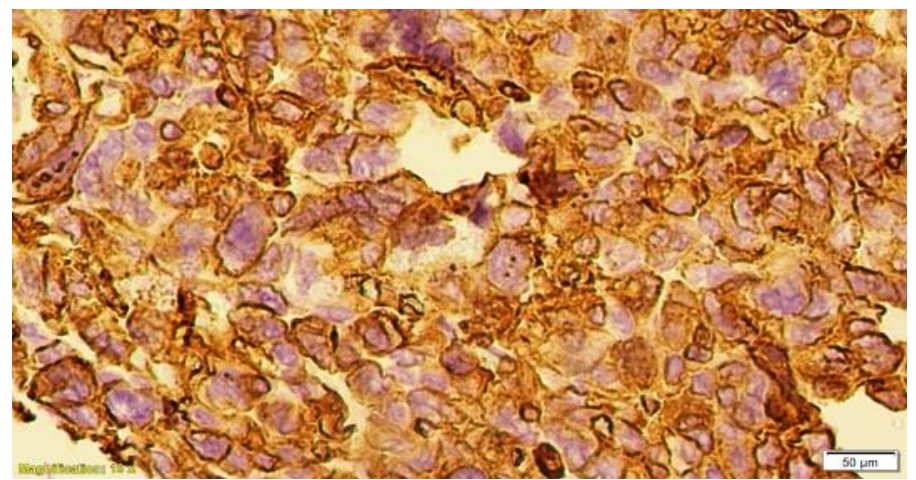

Fig. (10): HGSOC. Positive Vimentin cytoplasmic staining in $>30 \%$ of tumor cells. (Immunohistochemistry (IHC) X 200). 